\title{
Contralaterally transplanted human embryonic stem cell-derived neural precursor cells (ENStem-A) migrate and improve brain functions in stroke-damaged rats
}

\author{
Da-Jeong Chang ${ }^{1,6}$, Seung-Hun $\mathrm{Oh}^{2,6}$, Nayeon Lee ${ }^{1}$, Chunggab Choi ${ }^{1}$, Iksoo Jeon ${ }^{1}$, Hyun Sook Kim ${ }^{2}$, \\ Dong Ah Shin ${ }^{3}$, Seo Eun Lee ${ }^{4}$, Daehong Kim ${ }^{5}$ and Jihwan Song ${ }^{1,2}$
}

The transplantation of neural precursor cells (NPCs) is known to be a promising approach to ameliorating behavioral deficits after stroke in a rodent model of middle cerebral artery occlusion (MCAo). Previous studies have shown that transplanted NPCs migrate toward the infarct region, survive and differentiate into mature neurons to some extent. However, the spatiotemporal dynamics of NPC migration following transplantation into stroke animals have yet to be elucidated. In this study, we investigated the fates of human embryonic stem cell (hESC)-derived NPCs (ENStem-A) for 8 weeks following transplantation into the side contralateral to the infarct region using 7.0T animal magnetic resonance imaging (MRI). T2- and T2*-weighted MRI analyses indicated that the migrating cells were clearly detectable at the infarct boundary zone by 1 week, and the intensity of the MRI signals robustly increased within 4 weeks after transplantation. Afterwards, the signals were slightly increased or unchanged. At 8 weeks, we performed Prussian blue staining and immunohistochemical staining using humanspecific markers, and found that high percentages of transplanted cells migrated to the infarct boundary. Most of these cells were CXCR4-positive. We also observed that the migrating cells expressed markers for various stages of neural differentiation, including Nestin, Tuj1, NeuN, TH, DARPP-32 and SV38, indicating that the transplanted cells may partially contribute to the reconstruction of the damaged neural tissues after stroke. Interestingly, we found that the extent of gliosis (glial fibrillary acidic protein-positive cells) and apoptosis (TUNEL-positive cells) were significantly decreased in the cell-transplanted group, suggesting that hESC-NPCs have a positive role in reducing glia scar formation and cell death after stroke. No tumors formed in our study. We also performed various behavioral tests, including rotarod, stepping and modified neurological severity score tests, and found that the transplanted animals exhibited significant improvements in sensorimotor functions during the 8 weeks after transplantation. Taken together, these results strongly suggest that hESC-NPCs have the capacity to migrate to the infarct region, form neural tissues efficiently and contribute to behavioral recovery in a rodent model of ischemic stroke.

Experimental \& Molecular Medicine (2013) 45, e53; doi:10.1038/emm.2013.93; published online 15 November 2013

Keywords: behavioral recovery; human embryonic stem cell-derived neural precursor cells (hESC-NPCs, ENStem-A); magnetic resonance imaging (MRI); migration; stroke

\section{INTRODUCTION}

Stroke is one of the leading causes of death and disability worldwide. Although thrombolytic treatments, such as tissue plasminogen activator, are effective for the treatment of stroke, $<10 \%$ of stroke patients benefit from this type of treatment because of its narrow therapeutic time window (within $4.5 \mathrm{~h}$ after onset). ${ }^{1}$ Despite extensive research using animal models, no neuroprotective agent has been demonstrated to be effective in the recovery of primary or secondary damage following stroke in clinical trials. ${ }^{2}$

\footnotetext{
${ }^{1}$ Department of Biomedical Science, CHA Stem Cell Institute, CHA University, Seoul, Republic of Korea; ${ }^{2}$ Department of Neurology, Bundang CHA General Hospital, CHA University, Gyeonggi-do, Republic of Korea; ${ }^{3}$ Department of Neurosurgery, Yonsei University College of Medicine, Seoul, Republic of Korea; ${ }^{4}$ Department of Physiology, College of Medicine, Hanyang University, Seoul, Republic of Korea and ${ }^{5}$ Molecular Imaging and Therapy Branch, National Cancer Center, Gyeonggi-do, Republic of Korea

${ }^{6}$ These authors contributed equally to this work.

Correspondence: Professor J Song, CHA Stem Cell Institute, CHA University, 606-16 Yeoksam 1-dong, Kangnam-gu, Seoul 135-081, Republic of Korea. E-mail: jsong@cha.ac.kr

or Dr D Kim, Molecular Imaging and Therapy Branch, National Cancer Center, 809 Madu 1-dong, Ilsandong-gu, Goyangsi, Gyeonggi-do 410-769, Republic of Korea.

E-mail: dkim@ncc.re.kr
}

Received 30 June 2013; revised 23 July 2013; accepted 25 July 2013 
In animal models of stroke, the transplantation of neural precursor cells (NPCs) has been shown to be a promising approach to ameliorate behavioral deficits. Recently, the first clinical trial using NPCs in stroke patients was performed by ReNeuron using clonal, conditionally immortalized neural stem cells isolated from human fetal cortex, and the preliminary report suggests that the NPC transplantation is safe. ${ }^{3}$ Most previous experimental studies have indicated that NPCs transplanted into the ipsilesional cerebral hemisphere before or after stroke underwent robust targeted transparenchymal migration toward the lesion boundary. ${ }^{4-7}$ This intriguing phenomenon may be orchestrated by multiple factors or the host environment, which is not fully understood. Several studies have also shown that embryonic stem cells or NPCs that were transplanted into the side contralateral to the cerebral infarction also migrate toward the lesion and survive. $^{8-10}$ This result indicates that a strong, long-range chemotactic gradient sufficient to induce travel from the implantation site to the lesion site may be present.

In vivo cellular imaging techniques, such as magnetic resonance imaging (MRI) of supraparamagnetic iron oxide particle-labeled stem cells, enable the analysis of stem cell migration over the long periods of time in the same subject following transplantation. ${ }^{11,12}$ Unlike conventional end-point histology, in vivo MRI offers an opportunity to longitudinally monitor the implanted cells in vivo, allowing a direct assessment of the spatiotemporal dynamics of cell migration and survival following transplantation. However, there have been only a few studies regarding the long-term dynamic process of NPC migration using in vivo animal MRI.

In this study, we used a 7.0T animal MRI to track the fates of human embryonic stem cell (hESC)-derived NPCs (ENStem-A) labeled with ferumoxides (Feridex)-protamine sulfate complex following transplantation into a rodent stroke model up to 8 weeks. In addition, we evaluated whether the migrating NPCs to the lesion site can survive, differentiate into various types of neurons and contribute to functional improvement in a rodent model of ischemic stroke.

\section{MATERIALS AND METHODS}

Culture and characterization of hESC-NPCs (ENStem-A)

For this study, we purchased the hESC-NPC line, ENStem-A, from Millipore (Billerica, MA, USA). ENStem-A is a neural progenitor cell line derived from H9 hESCs. The ENStem-A cells were cultured according to the manufacturer's instructions. Briefly, they were incubated in ENStem-A Neural Expansion Medium (Chemicon, Temecula, CA, USA) supplemented with fibroblast growth factor-2 $\left(20 \mathrm{ng} \mathrm{ml}^{-1}\right)$. To analyze the in vitro marker expression of ENStem-A cells, we performed immunocytochemical analyses using the following primary antibodies: SOX2 (1:200, Chemicon), neural cell adhesion molecule (1:500, Chemicon), human-specific Nestin (1:250, R\&D Systems, Minneapolis, MN, USA) and Musashi (1:500, Chemicon). The secondary antibodies used were Alexa 555-conjugated goat anti-mouse IgG (1:200, Molecular Probes, Eugene, OR, USA) and Alexa 488-conjugated goat anti-rabbit IgG (1:200, Molecular Probes). The staining patterns were examined and photographed using a confocal laser scanning microscope imaging system (LSM510, Carl Zeiss, Inc.,Thornwood, NY, USA).
For reverse transcription-PCR analysis, we isolated total RNA from cells using the TRIzol RNA extraction method (Gibco, Gaithersburg, MD, USA) and synthesized complementary DNA using M-MLV reverse transcriptase (Promega, Madison, WI, USA) at $42^{\circ} \mathrm{C}$ for $1 \mathrm{~h}$. PCR amplification was performed using Taq polymerase according to the manufacturer's instructions (Intron Biotechnology, Gyeonggi-do, Korea). Below is the following information for the primers used in this study: sequences, GenBank accession numbers, expected product sizes, and annealing temperatures. All PCR reactions included 30 cycles of amplification.

Sox2 (NM_003106): forward (F): 5'-GCTGCAAAAGA GAACACCAA-3', reverse (R): 5'-CTTCCTGCAAAGCTCCTACC-3' (232 bp, 59 $\left.{ }^{\circ} \mathrm{C}\right)$; Nestin (NM_006617): F: $5^{\prime}$-TCCAGAAACTCAAGCA CCA-3', R: $5^{\prime}$-AAATTCTCCAGGTTCCATGC-3' (183 bp, $\left.59^{\circ} \mathrm{C}\right)$; III $\beta$ tubulin (BC000748.2): F: 5'-ATGAGGGAGATCGTGCACAT-3', R: 5' GCCCCTGAGCGGACACTGT-3' (239 bp, $59^{\circ} \mathrm{C}$ ); Map2 (U01828): F: 5'-GCATATGCGCTGATTCTTCA-3'， R: 5'-CTTTCCGTTCATCTG CCATT-3' (202 bp, $\left.59^{\circ} \mathrm{C}\right)$; GFAP (BC041765.1): F: 5'-GCAGAGAT GATGGAGCTCAATGACC-3'， R: 5'-GTTTCATCCTGGAGCTTCT GCCTCA-3' (266 bp, $59^{\circ} \mathrm{C}$ ); and GAPDH (BC083511.1): F: 5'GTCATACCAGGAAATGAGCT-3'， R: 5'-TGACCACAGTCCATGCCATC-3' $\left(422 \mathrm{bp}, 60^{\circ} \mathrm{C}\right)$.

\section{Generation of the rodent MCAo model and cell transplantation}

The manipulation of the experimental animals was performed in accordance with the guidelines of the CHA University IACUC (Institutional Animal Care and Use Committee; IACUC090012). Adult male Sprague-Dawley rats (Orient, Seoul, Korea) weighing 270 to $300 \mathrm{~g}$ were used in this study. The rats were housed with access to food and water ad libitum under a 12-h light/dark cycle. After the rats had been anesthetized with $1 \%$ ketamine $\left(30 \mathrm{mg} \mathrm{kg}^{-1}\right.$, intraperitoneal) and xylazine hydrochloride $\left(4 \mathrm{mg} \mathrm{kg}^{-1}\right.$, intraperitoneal), we maintained the rats' body temperatures at $37 \pm 1{ }^{\circ} \mathrm{C}$ using a rectal probe and a heating pad. To induce middle cerebral artery occlusion (MCAo), we used the method of Longa et al. ${ }^{13} \mathrm{~A}$ blunt-ended monofilament (4-0, Ethicon, Livingston, Scotland, UK) was inserted into the internal carotid artery from the bifurcation to occlude the middle cerebral artery. The monofilament was carefully removed $90 \mathrm{~min}$ after MCAo induction.

On the next day, we assessed the acute neurological severity using circling behavior and forelimb/hindlimb placement tests to select the appropriate stroke animals. For transplantation, animals were divided into the transplantation group, which received $2 \times 10^{5} \mathrm{hESC}-\mathrm{NPCs}$ (ENStem-A) in a total volume of $2 \mu \mathrm{l}(n=8)$, and the sham group, which received $2 \mu \mathrm{l}$ of culture medium $(n=6)$. At 1 week after MCAo induction, ENStem-A cells were transplanted into the contralateral side in the striatum region using the following stereotaxic coordinates: $1.0 \mathrm{~mm}$ anteroposterior, $3.0 \mathrm{~mm}$ mediolateral and $5.0 \mathrm{~mm}$ dorsoventral from the bregma. The 26-gauge Hamilton syringe (Reno, NV, USA) was left in place for an additional $5 \mathrm{~min}$ to stabilize the transplanted cells. All rats received cyclosporine A (Sigma, St Louis, MO, USA, $10 \mathrm{mg} \mathrm{kg}^{-1}$, intraperitoneal) for immunosuppression from 1 day before transplantation until the end of the observation period for 8 weeks after transplantation.

\section{Behavioral tests}

All animals were trained under the same conditions three times a day for three consecutive days before the induction of MCAo to minimize variation among the animals. In the rotarod test, in which the rod 
speed progressively increased from 4 to 40 r.p.m. for $2 \mathrm{~min}$, all rats were placed on the rotarod cylinder, and the time that each rat remained on the cylinder was recorded. Each week for 8 weeks after transplantation, we recorded the time when the animals fell off the rotating rod, and we calculated the average time (in seconds) for three trials. In the stepping test, all rats were initially familiarized with the experimenter's grip. The number of forepaw placements was counted for each rat to assess akinesia, when they were moved slowly in both forehand and backhand directions along the edge of a table over a distance of $90 \mathrm{~cm}$ in $5 \mathrm{sec}$. All animals were examined weekly for 8 weeks. We also performed the modified neurological severity score test, the scores of which were interpreted as follows: 0 point, normal; 1-9 points, mild injury; 10-19 points, moderate injury; 20-27 points, severe injury; 28 points, the most severe condition.

\section{MRI detection of the labeled cells}

To label the cells for animal MRI, we used Feridex (Taejoon Pharm, Seoul, Korea) as a contrast agent. The Feridex-protamine sulfate complex was prepared at a concentration of $2 \mu \mathrm{g} \mathrm{ml}^{-1}$ in culture medium without serum, and this mixture was mixed by hand shaking and incubated for $30 \mathrm{~min}$ at room temperature. Then, an equal volume of culture medium and the Feridex-protamine sulfate complex was added to the cells, and they were incubated for $12-16 \mathrm{~h}$ at $37^{\circ} \mathrm{C}$. The medium was removed, and the cells were washed twice with phosphate-buffered saline (PBS). All rats were examined to determine whether the Feridex-labeled ENStem-A cells migrated toward the ischemic region using a 7.0T MRI (BioSpec 70/20, Bruker, Germany). The cell locations were assessed for $\sim 8$ weeks following transplantation. T2-weighted imaging was used to assess the full extent of the infarct, and $\mathrm{T}^{\star}$ imaging was used to track the location and migration of the labeled cells. At 8 weeks after transplantation, we performed Prussian blue staining to detect the Feridex-labeled cells and to track the contralaterally transplanted stem cells in the ischemic brain.

\section{Immunohistochemical analysis}

At 8 weeks after transplantation, the rats were transcardially perfused with heparinized saline $(0.9 \% \mathrm{NaCl})$. The brains were fixed in $4 \%$ paraformaldehyde at room temperature overnight, transferred to $30 \%$ sucrose for 1-3 days at $4{ }^{\circ} \mathrm{C}$, and then embedded in optimal cutting temperature compound (OCT, Sakura Finetek, Tokyo, Japan). Doublelabel immunofluorescence staining was performed on free-floating $40 \mu \mathrm{m}$-thick sections. Sections were incubated in blocking solution, which consisted of 5\% normal horse serum (Vector Laboratories, Burlingame, CA, USA) in PBS containing 0.3\% Triton X-100 (Sigma) for $1 \mathrm{~h}$ at room temperature. The following primary antibodies were used: human-specific nuclei ( $\mathrm{hNu}$ ) (1:100, Chemicon), human-specific mitochondria (hMito) (1:250, Chemicon), human-specific Nestin (1:250, R\&D Systems), NeuN (1:500, Millipore), DARPP-32 (1:100, Cell Signaling Technology, Danvers, MA, USA), tyrosine hydroxylase (TH) (1:1000, Pel-Freez, Rodgers, AR, USA), synaptophysin (SVP-38) (1:200, Sigma), and CXCR4 (1:40, R\&D Systems). After several washes in PBS, the brain sections were incubated with the following secondary antibodies for $2 \mathrm{~h}$ at room temperature: Alexa 488 - or 555-conjugated goat anti-mouse IgG or Alexa 488- or 555-conjugated goat anti-rabbit IgG. Next, the sections were counterstained with the nuclear marker $4^{\prime}, 6^{\prime}$-diamidino-2-phenylindole (DAPI).

To detect the Feridex-labeled cells in vivo, Prussian staining was performed. Tissue sections were fixed in methanol for $30 \mathrm{~min}$ at room temperature, followed by treatment with a mixture of $4 \%$ potassium ferrocyanide and $4 \% \mathrm{HCl}$ solution. After boiling, these sections were washed with PBS, mounted and examined under a light microscope (Eclipse E600, Nikon, Tokyo, Japan). To detect apoptotic cells, TUNEL (terminal deoxynucleotidyl transferase-mediated dUTP nick-end labeling) assay was performed. Frozen sections were cut and fixed in $4 \%$ paraformaldehyde in PBS for $20 \mathrm{~min}$ at room temperature. After three washes in PBS for $30 \mathrm{~min}$, the sections were stained using the In Situ Cell Death Detection Kit (Roche, Indianapolis, IN, USA) according to the manufacturer's instructions. All sections were counterstained with $4^{\prime}, 6^{\prime}$-diamidino-2-phenylindole (DAB). The number of TUNEL-positive cells per field in the vicinity of the infarct region was quantified using ImageJ software (NIH, Bethesda, MD, USA).

\section{Statistical analysis}

Statistical analysis was performed using the Statistical Analysis System program (Enterprise 4.1; SAS, Seoul, Korea). All values are presented as the mean \pm s.e.m. (standard error of mean). The statistical significance of the differences between the two groups for the behavioral scores was analyzed by one-way analysis of variance (ANOVA). A probability value of $<0.05$ was considered significant.

\section{RESULTS}

\section{Characterization of hESC-NPCs (ENStem-A)}

To characterize hESC-NPCs (ENStem-A), we plated them on a coverslip precoated with laminin. The ENStem-A cells proliferated as an adherent monolayer. We found that they robustly express Sox2 (neuroepithelial cell marker), neural cell adhesion molecule (neural progenitor marker), Nestin (neural stem marker) and Musashi (neural progenitor marker) (Figures 1a-d). We also observed that the ENStem-A cells expressed transcripts for neural precursor markers, such as Sox 2 and Nestin, and an early neural marker, type III- $\beta$ tubulin (III $\beta$-tub), whereas there was no expression of markers of mature neurons, such as microtubuleassociated protein 2 (Map2), or glial cells, such as glial fibrillary acidic protein (GFAP) (Figure 1e). These results indicate that ENStem-A cells represent a homogeneous population of NPCs.

\section{MRI tracking of contralaterally transplanted hESC-NPCs (ENStem-A) in vivo}

To track the location and migration of transplanted hESCNPCs in vivo, we intracerebrally transplanted ENStem-A cells into the striatum on the side contralateral to the ischemic injury. Figure 2a shows representative $\mathrm{T} 2{ }^{*}$-weighted $\mathrm{MR}$ images taken before cell transplantation and at 5, 7, 25, 32, 39, 46 and 53 days after cell transplantation. Using a sequential series analysis, we examined the distribution of migrated cells from $2.76 \mathrm{~mm}$ anterior to $-4.36 \mathrm{~mm}$ posterior to the landmark position of the bregma (Figure 2a). As early as 5 days after transplantation into the hemisphere contralateral to the infarct area, we were able to detect hypointense signals, indicative of migrating cells, in the ipsilateral side in the $\mathrm{T} 2^{*}$-weighted images for all tested animals. The area of the cell migration signals on the MR images exhibited a robust increase from 7 days to 25 days after transplantation in most of the examined animals, and afterwards, no change in the intensity of the cell migration signals was found up to 53 days after transplantation. The migrating cells were primarily found at the boundary 

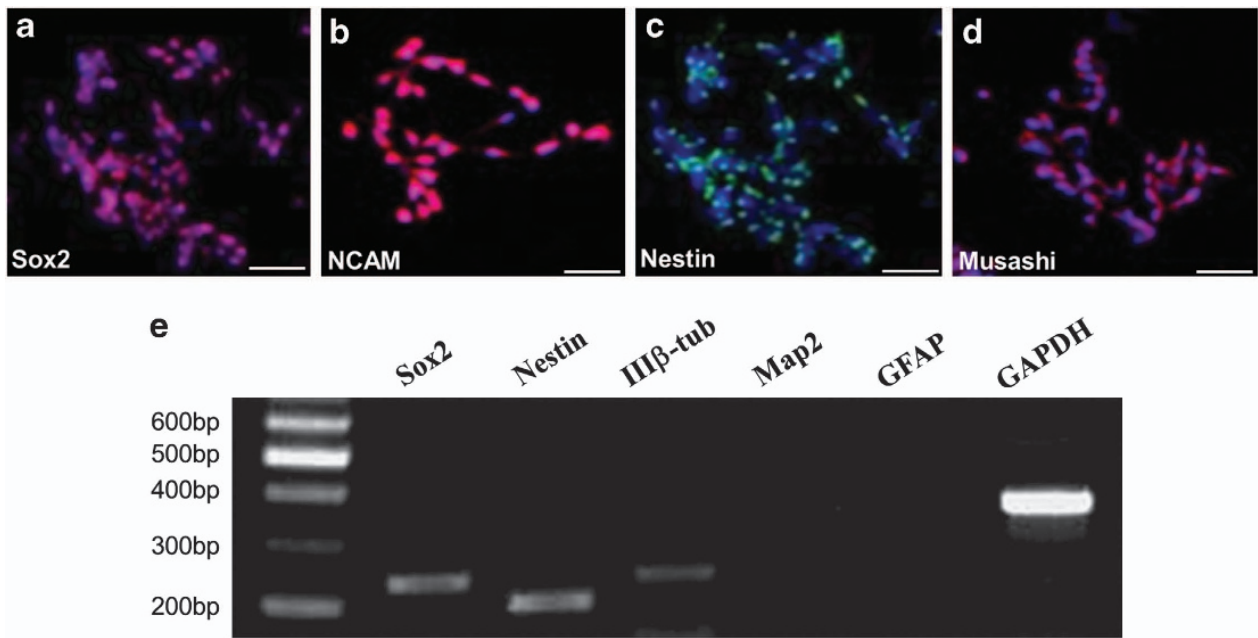

Figure 1 Characteristics of the human embryonic stem cell-derived neural precursor cells (hESC-NPCs, ENStem-A) used in this study. Immunocytochemical staining showing the expression of neural stem/progenitor makers: (a) Sox2, (b) neural cell adhesion molecule (NCAM), (c) Nestin and (d) Musashi. The nuclei were counterstained with 4',6'-diamidino-2-phenylindole (DAPI; blue). Scale bar, $20 \mu \mathrm{m}$. (e) Reverse transcription-PCR (RT-PCR) analysis showing that ENStem-A cells express high levels of Sox2 and Nestin and low levels of type III- $\beta$ tubulin (III $\beta$-tub). The expression of microtubule-associated protein 2 (Map2) and glial fibrillary acidic protein (GFAP) was not detectable.

of the infarct lesion (from $1.68 \mathrm{~mm}$ anterior to $-0.48 \mathrm{~mm}$ posterior to the bregma), but no signals were detected in the infarct core, corpus callosum or other cerebral areas by MRI.

After the completion of the in vivo MRI study, the animals were transcardially perfused, and histological evaluation was performed. We first performed Prussian blue staining on the brain sections 53 days after transplantation ( $\sim 2$ months after the stroke) to determine whether the migrating cells detected by MRI were the transplanted cells. The Prussian blue staining specifically detected Feridex-labeled cells at the infarct borders, where positive cell migration signals were also detected by T2*-weighted MRI (Figure 2b). In a double-staining confocal analysis using antibodies against hMito and CXCR4, a chemokine receptor that recognizes inflammatory signals and other signals, we observed that hMito-positive migrating cells were colocalized with CXCR4 at the infarct border (Figure 2c). We also stained the brain sections with $\mathrm{hNu}$ antibody to determine the number of surviving cells, and then counted the number of hNu-positive cells using unbiased stereology. These cells were located primarily in the peri-infarct area of the adjacent striatum, and some hNu-positive cells were also detected in the corpus callosum (data not shown). The detected $\mathrm{hNu}$ positive cells were estimated to represent $\sim 20 \%$ $(\sim 19.97 \pm 13.98 \%)$ of the initial number of transplanted cells.

\section{Neuronal differentiation of transplanted hESC-NPCs (ENStem-A) in vivo}

We observed that a large percentage of the transplanted ENStem-A cells that migrated toward the infarct border zone were still hNestin-positive neural precursor/stem cells (Figure 3a). The hMito- or hNu-positive cells were found along the border of the ischemic region. Interestingly, Tuj1positive and hMito-positive cells were found in the ischemic cortex region (Figure $3 \mathrm{~b}$ ). The hMito-positive cells were also shown to be colocalized with NeuN, a marker of mature neurons (Figure 3c). We also found that small numbers of the transplanted cells had differentiated into neurons positive for TH (Figure 3d) or DARPP-32 (Figure 3e) at the peri-infarct region of the lateral striatum. The hMito-positive cells were also colocalized with SVP38, a marker for synaptic vesicle protein, in the peri-infarct region (Figure 3f).

\section{Functional outcomes of MCAo animals after hESC-NPC transplantation}

All behavior tests were conducted with both the hESC-NPC (ENStem-A)-transplanted group and the sham group from postoperative day 3 to 8 weeks after transplantation. There was no significant difference in the scores for the behavior tests between the ENStem-A and Sham groups after MCAo induction. In the rotarod test, MCAo animal models showed severe impairment. In the ENStem-A group, transplanted animals showed a slight increase 2 weeks after transplantation compared with the sham group, and this improvement gradually became more prominent from 3 weeks $(P<0.05)$ to 8 weeks $(P<0.001)$ after transplantation (Figure $4 a)$. In the stepping test, the ENStem-A group exhibited significantly improved performance compared with the sham group only 7 weeks and 8 weeks after transplantation $(P<0.001$, Figure $4 \mathrm{~b})$. In the modified neurological severity score test, we observed that the ENStem-A group improved by 1 week after transplantation compared with the sham group $(P<0.05)$, and the extent of this improvement became more significant between 4 and 8 weeks after transplantation $(P<0.001$; Figure $4 \mathrm{c})$.

\section{Reduction of gliosis and apoptosis by transplanted hESC-NPCs (ENStem-A)}

We measured the number of GFAP-positive cells in the periinfarct area 8 weeks after transplantation. The ENStem-A 

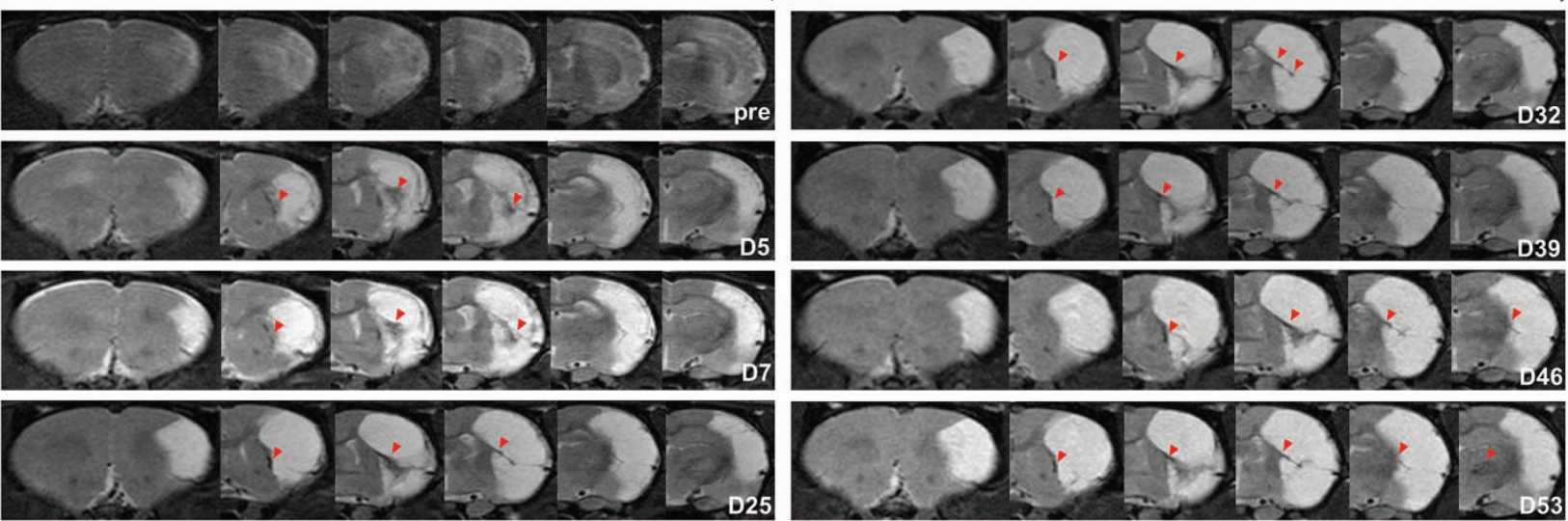

b
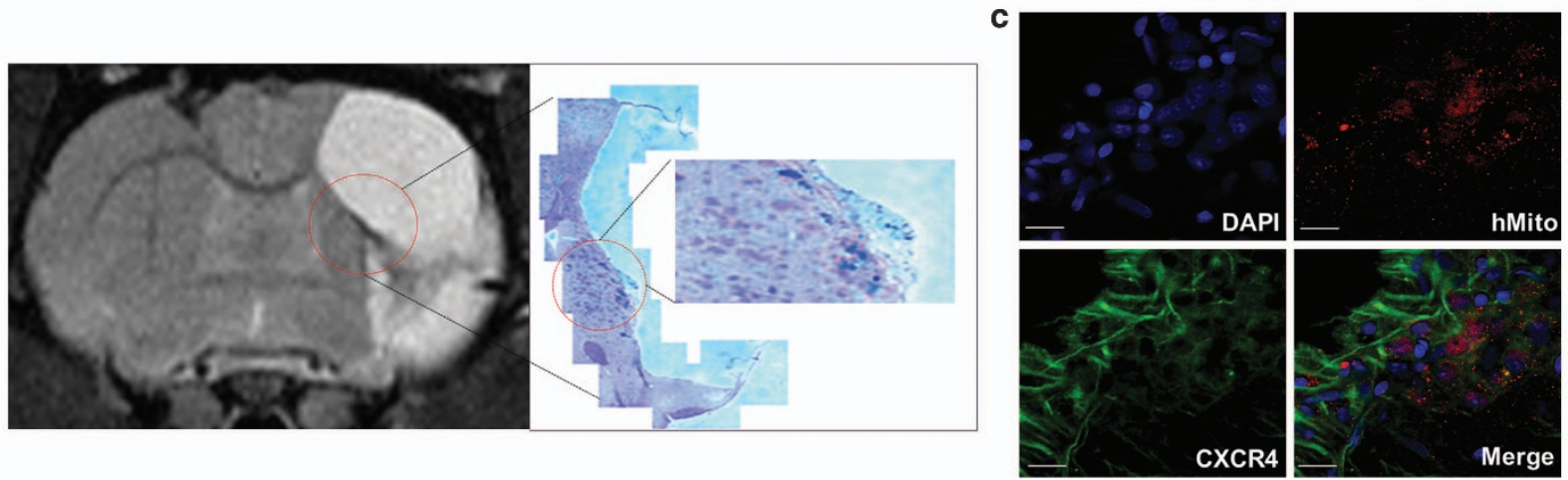

Figure 2 Detection and visualization of Feridex-labeled human embryonic stem cell-derived neural precursor cells (hESC-NPCs, ENStem-A) using 7.0T animal magnetic resonance imaging (MRI) and histological methods. (a) Serial T2*-weighted MR images showing the migration and distribution of the contralaterally transplanted cells. MR images were taken from 5 days to 53 days following transplantation. The red arrows indicate the cells migrating toward the ischemic peri-infarct region. (b) Hyperintense region in the T2*-weighted MR image indicating the location of transplanted cells (left) and the corresponding Prussian blue staining, confirming the presence of transplanted Feridex-labeled cells (shown in blue) (right). (c) Confocal analysis showing that human-specific mitochondria (hMito)-positive migrating human cells express the chemokine receptor CXCR4. DAPI (4',6'-diamidino-2-phenylindole) was used to counterstain the cells. Scale bar, $20 \mu \mathrm{m}$.

group had a significantly lower number of GFAP-positive cells in the peri-infarct area compared with the sham group $(29.2 \pm 4.4 \%$ vs $79.2 \pm 4.8 \% ; P<0.01 ;$ Figures $5 \mathrm{a}-\mathrm{c})$. The number of TUNEL-positive cells in the peri-infarct area was also significantly reduced in the ENStem-A group compared with the sham group $(5.6 \pm 1.3 \%$ vs $23.5 \pm 1.7 \% ; P<0.01$; Figures $5 \mathrm{~d}-\mathrm{f}$ ). Between the transplanted group and the sham group, there were no significant differences in the expression levels of ED1 (microglia and macrophage maker; $28.4 \pm 1.5 \%$ vs $23.8 \pm 2.7 \% ; P=0.58$ ) and Iba-1 (activated microglia marker; $26.7 \pm 0.6 \%$ vs $14.9 \pm 1.1 \% ; P=0.31$; data not shown).

\section{DISCUSSION}

In the present study, we observed the pronounced migration of hESC-NPCs (ENStem-A) toward the injured site in a rodent model of ischemic stroke using a serial in vivo animal MRI analysis over a period of 53 days. The contralaterally transplanted cells were detected in the injured brain region, primarily in the ischemic boundary zone, as early as 5 days after transplantation, and the migration signals robustly increased within $\sim 1$ month after transplantation. This finding was consistent with those of previous MRI studies on contralaterally transplanted NPCs. ${ }^{9}, 10$ This finding is also in agreement with our previous study, in which extensive migration of human bone marrow-derived mesenchymal stem cells toward the infarct area was detected. ${ }^{11}$ It has also been shown that even systemically administered NPCs can home toward the infarct area via an unknown mechanism. ${ }^{14}$ It is conceivable that there could be multiple factors confounding the real signals that are solely derived from labeled cells in a strokedamaged brain, among which hemorrhage is the most important factor.

In our study, no hemorrhagic lesions were found in the histological analysis, and numerous hNu-positive cells were detected in the infarct boundary up to 8 weeks after transplantation. We also found numerous Prussian blue-positive cells in the corresponding area, where hypointense signals were predominantly detected by MRI analysis. An additional confounder is the transport of dead Feridex-labeled cells by macrophages, which could be misinterpreted as cell migration. ${ }^{15}$ In this study, we did not quantify the Feridex-labeled cells phagocytosed by inflammatory cells. However, previous 

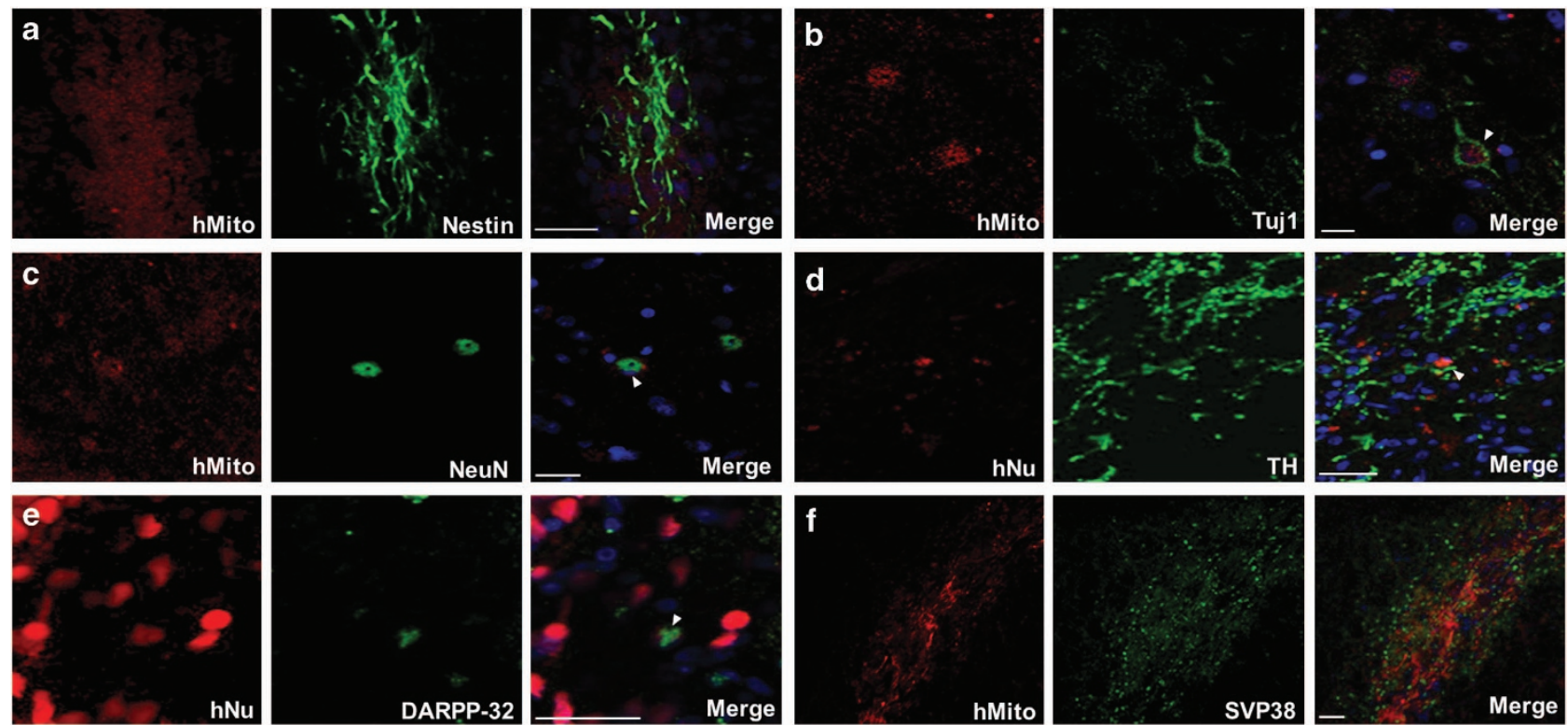

Figure 3 Immunohistochemical analyses showing the extent of neuronal differentiation 8 weeks following the transplantation of ENStem-A cells into middle cerebral artery occlusion (MCAo)-induced rats. Transplanted human cells were identified using antibodies against humanspecific nuclei (hNu) or human-specific mitochondria (hMito), which are shown in red. Transplanted cells were shown to express markers of neural stem/progenitor cells (Nestin; a) and early and general neurons (type III- $\beta$ tubulin (Tuj1; b), as well as a neuron-specific nuclear protein, NeuN (c). They also expressed markers of dopaminergic neurons (tyrosine hydroxylase (TH; d), and medium spiny neurons (dopamine- and cAMP-regulated neuronal phosphoprotein-32 (DARPP-32; e). Some of the transplanted cells were positive for a synaptic vesicle protein, SVP38 (f). Scale bar, $50 \mu \mathrm{m}$.
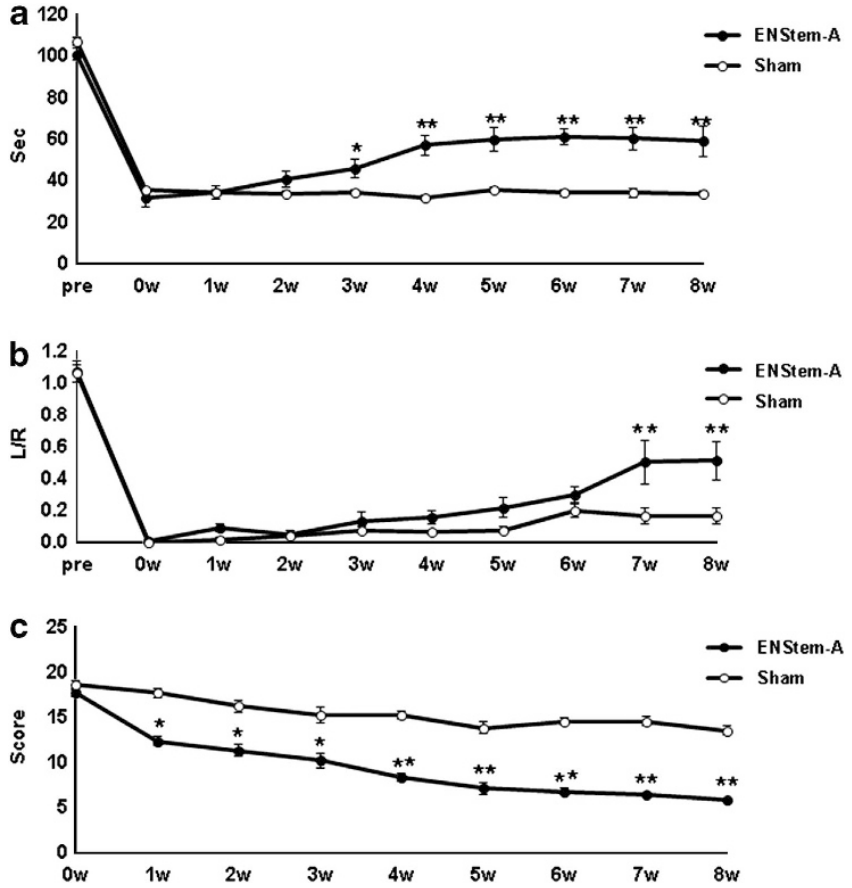

Figure 4 Behavioral improvement following the transplantation of ENStem-A cells into middle cerebral artery occlusion (MCAo)induced rats. Scores from the rotarod test (a), stepping test (b) and modified neurological severity score (mNSS) test (c) are shown for the 8-week study period. MCAo was induced at week 0 . The data are presented as the mean values \pm s.e.m. The numbers of animals used in the ENStem-A group and the sham group were 8 and 6 , respectively. ${ }^{*} P<0.05$ and ${ }^{* *} P<0.001$. studies on the contralaterally transplanted fetal NPCs in stroke models demonstrated that the majority of supraparamagnetic iron oxide particle-labeled cells (85-90\%) were Iba-1-negative at the infarct boundary zone. ${ }^{10}$ This result suggests that the number of migrating cells that were phagocytosed by inflammatory cells was relatively low. Even in the most extreme case, some of the transplanted cells can indeed migrate toward the injured site.

In this study, we demonstrated that the transplanted cells exhibited a clear directional migration toward the lesion area using serial MRI analysis. Given the long-distance directed migration from the transplantation site to the lesion, it has been postulated that there exists a strong, long-range chemotactic signal with a concentration gradient emanating from the lesion. ${ }^{9}$ NPCs possess specific receptors that allow them to sense gradients of attractive signals generated by the injury site and to migrate toward the lesion. There is increasing evidence that chemokines such as stromal cell-derived factor 1 (SDF-1) regulate the NPC migration after central nervous system injury and inflammation. Numerous studies have shown that the interaction of CXCR4, an SDF-1 receptor, with SDF-1 could play an important role in neural progenitor cell migration. ${ }^{16}$ Previous in vitro and in vivo studies have demonstrated that neural progenitor cells express CXCR4 and migrate toward a source of SDF1, and CXCR4 was expressed by the transplanted human NPCs migrating to the injured area in the rodent stroke model. ${ }^{4,17}$ In the present study, we were also able to detect CXCR4-positive cells among the migrating cells at the infarct boundary zone when human embryonic stem cellderived NPCs (ENStem-A) were transplanted contralaterally. 

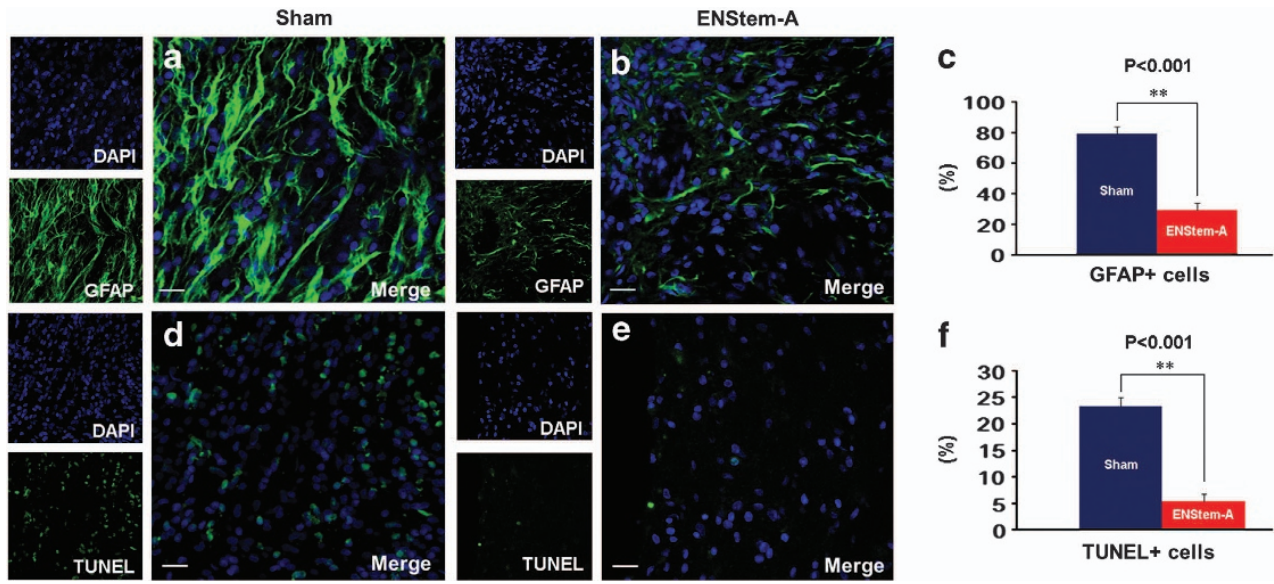

Figure 5 Histological analyses on the extents of gliosis and apoptosis following cell transplantation into middle cerebral artery occlusion (MCAo)-induced rats. The transplanted group (ENStem-A) exhibited fewer numbers of glial fibrillary acidic protein (GFAP)-positive and terminal deoxynucleotidyl transferase-mediated dUTP nick-end labeling (TUNEL)-positive cells in the peri-infarct region 8 weeks after MCAo compared with the sham group (a-f). Scale bar, $20 \mu \mathrm{m}$. The data are presented as the mean values \pm s.e.m. ${ }^{* *} P<0.001$.

The exact molecular mechanisms involved in this chemotaxis or tropism have yet to be elucidated.

Notably, we observed significant functional improvement following the transplantation of hESC-NPCs (ENStem-A). The gradual sensorimotor improvement over several weeks after cell transplantation suggests that the NPCs have a true recovery-enhancing effect after stroke. In the current study, $\sim 20 \%$ of the transplanted cells were detected at the injured site in the brain, and only a small proportion of the transplanted cells expressed various types of mature neuronal markers. Some of the transplanted cells were colocalized with SVP38, a synaptic vesicle protein, at the infarct boundary zone, indicating the formation of synapses by the transplanted cells. It is likely that the functional improvement observed in this study was mediated by cell replacement by the grafted stem cells to a certain extent, a hypothesis that is supported by a previous study in which grafted mouse ESC-NPCs differentiated into mature neurons, as demonstrated by immunohistochemistry and patch clamp studies conducted 12 weeks after transplantation. ${ }^{5}$ However, we cannot exclude the possibility that the cell replacement effect is not the sole mechanism responsible for the observed functional improvement after NPC transplantation, primarily because the extent of differentiation of the transplanted cells into mature neurons was not sufficiently high to convincingly explain the observed effects. Furthermore, there was no significant change in the infarct size after transplantation. Therefore, it is conceivable that the transplanted cells ameliorated the host environment in the damaged area via the 'bystander effect'.

Many previous studies have demonstrated that NPCs exhibit anti-inflammatory, anti-apoptotic or glial scar-inhibitory effects after brain injury, thereby enhancing functional recovery. ${ }^{18,19}$ In support of this notion, the systemic administration of NPCs has been reported to decrease inflammation, gliosis and apoptosis following stroke, despite the extremely low number of cells $(<2 \%)$ engrafted in the host brain. ${ }^{14}$ In the present study, we also observed that the

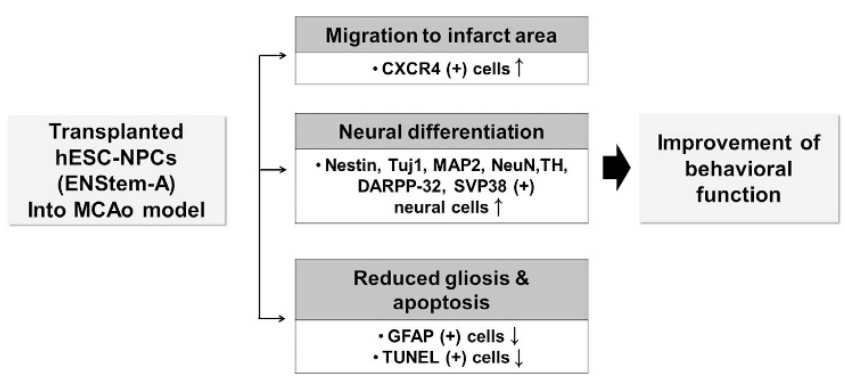

Figure $6 \mathrm{~A}$ proposed mode of action of the transplanted human embryonic stem cell-derived neural precursor cells (hESC-NPCs, ENStem-A) in a rodent model of ischemic stroke.

numbers of GFAP-positive and TUNEL-positive cells were significantly lower in the NPC-treated rats, suggesting that the observed functional recovery may have also been mediated by the anti-gliosis and anti-apoptotic effects of the transplanted NPCs. However, in the context of inflammation, we observed no significant differences in the numbers of ED1- or Iba-1positive cells between the NPC-treated and sham groups.

Brain inflammation, especially microglial activation, is an important process leading to secondary neuronal damage and scar formation following cerebral ischemia, depending on the time course of stroke. $^{20}$ Whether NPC transplantation increases or decreases microglial cell activation ${ }^{14,19,21,22}$ is still under debate because of the paucity of data and the differences in experimental design among the studies. Therefore, we cannot rule out the possibility that the negative results from our study are due to the fact that our histological examination was performed at a late stage ( 2 months), given that brain inflammation primarily occurs during the early phase after stroke (from several days to 3 weeks). ${ }^{23,24}$ Therefore, further study is necessary to determine the exact mechanisms responsible for the effects of hESC-NPCs on brain inflammation and endogenous neurogenesis, which is another important aspect of brain recovery, following stroke using different time courses. 
In conclusion, we demonstrated that contralaterally transplanted hESC-NPCs extensively migrate to and survive in the infarct region using a serial in vivo MRI analysis in a rodent model of ischemic stroke. Our study suggests that the long-term improvement of functional deficits after stroke may be mediated by neurorestorative (that is, neuronal differentiation and/or regeneration) and/or neuroprotective effects (that is, anti-scarring and anti-apoptotic effects) of the transplanted hESC-NPCs (Figure 6). Further studies on the detailed chemotactic mechanism of hESC-NPCs and optimization of the therapeutic conditions are necessary to develop more effective stem cell-based therapies for stroke in the clinical setting.

\section{CONFLICT OF INTEREST}

The authors declare no conflict of interest.

\section{ACKNOWLEDGEMENTS}

This study was supported by a grant from the Korea Healthcare Technology R\&D project, Ministry of Health, Welfare \& Family Affairs, Republic of Korea (A111016). JS is highly indebted to Professor Olle Lindvall in University of Lund, Sweden, who provided the initial support and the great inspiration on stroke research.

1 van Wijngaarden JD, Dirks M, Huijsman R, Niessen LW, Fabbricotti IN, Dippel DW. Hospital rates of thrombolysis for acute ischemic stroke: the influence of organizational culture. Stroke 2009; 40: 3390-3392.

2 Auriel E, Bornstein NM. Neuroprotection in acute ischemic stroke-current status. J Cell Mol Med 2010; 14: 2200-2202.

3 Mack GS. ReNeuron and StemCells get green light for neural stem cell trials. Nat Biotechnol 2011; 29: 95-97

4 Kelly S, Bliss TM, Shah AK, Sun GH, Ma M, Foo WC et al. Transplanted human fetal neural stem cells survive, migrate, and differentiate in ischemic rat cerebral cortex. Proc Natl Acad Sci USA 2004; 101 11839-11844.

5 Buhnemann C, Scholz A, Bernreuther C, Malik CY, Braun H, Schachner M et al. Neuronal differentiation of transplanted embryonic stem cell-derived precursors in stroke lesions of adult rats. Brain 2006; 129: 3238-3248.

6 Darsalia V, Kallur T, Kokaia Z. Survival, migration and neuronal differentiation of human fetal striatal and cortical neural stem cells grafted in strokedamaged rat striatum. Eur J Neurosci 2007; 26: 605-614.

7 Hayashi J, Takagi Y, Fukuda H, Imazato T, Nishimura M, Fujimoto M et al. Primate embryonic stem cell-derived neuronal progenitors transplanted into ischemic brain. J Cereb Blood Flow Metab 2006; 26: 906-914.

8 Kim DE, Schellingerhout D, Ishii K, Shah K, Weissleder R. Imaging of stem cell recruitment to ischemic infarcts in a murine model. Stroke 2004; 35: 952-957.

9 Hoehn M, Kustermann E, Blunk J, Wiedermann D, Trapp T, Wecker S et al. Monitoring of implanted stem cell migration in vivo: a highly resolved in vivo magnetic resonance imaging investigation of experimental stroke in rat. Proc Natl Acad Sci USA 2002; 99: 16267-16272.
10 Guzman R, Bliss T, De Los Angeles A, Moseley M, Palmer T, Steinberg G Neural progenitor cells transplanted into the uninjured brain undergo targeted migration after stroke onset. J Neurosci Res 2008; 86: 873-882.

11 Kim D, Chun BG, Kim YK, Lee YH, Park CS, Jeon I et al. In vivo tracking of human mesenchymal stem cells in experimental stroke. Cell Transplant 2008; 16: 1007-1012.

12 Pendharkar AV, Chua JY, Andres RH, Wang N, Gaeta X, Wang $\mathrm{H}$ et al. Biodistribution of neural stem cells after intravascular therapy for hypoxicischemia. Stroke 2010; 41: 2064-2070.

13 Longa EZ, Weinstein PR, Carlson S, Cummins R. Reversible middle cerebral artery occlusion without craniectomy in rats. Stroke 1989; 20: 84-91.

14 Bacigaluppi M, Pluchino S, Peruzzotti-Jametti L, Kilic E, Kilic U, Salani G et al. Delayed post-ischaemic neuroprotection following systemic neural stem cell transplantation involves multiple mechanisms. Brain 2009; 132: 2239-2251.

15 Rogers WJ, Basu P. Factors regulating macrophage endocytosis of nanoparticles: implications for targeted magnetic resonance plaque imaging. Atherosclerosis 2005; 178: 67-73.

16 Imitola J, Raddassi K, Park KI, Mueller FJ, Nieto M, Teng YD et al. Directed migration of neural stem cells to sites of CNS injury by the stromal cell-derived factor 1 alpha/CXC chemokine receptor 4 pathway. Proc Natl Acad Sci USA 2004; 101: 18117-18122.

17 Tran PB, Ren D, Veldhouse TJ, Miller RJ. Chemokine receptors are expressed widely by embryonic and adult neural progenitor cells. $J$ Neurosci Res 2004; 76: 20-34.

18 Lee HJ, Kim KS, Kim EJ, Choi HB, Lee KH, Park IH et al. Brain transplantation of immortalized human neural stem cells promotes functional recovery in mouse intracerebral hemorrhage stroke model. Stem Cells 2007; 25: 1204-1212.

19 Lee ST, Chu K, Jung KH, Kim SJ, Kim DH, Kang KM et al. Antiinflammatory mechanism of intravascular neural stem cell transplantation in haemorrhagic stroke. Brain 2008; 131: 616-629.

20 ladecola C, Anrather J. The immunology of stroke: from mechanisms to translation. Nat Med 2011; 17: 796-808.

21 Minnerup J, Kim JB, Schmidt A, Diederich K, Bauer H, Schilling M et al. Effects of neural progenitor cells on sensorimotor recovery and endogenous repair mechanisms after photothrombotic stroke. Stroke 2011; 42: 1757-1763.

22 Daadi MM, Davis AS, Arac A, Li Z, Maag AL, Bhatnagar R et al. Human neural stem cell grafts modify microglial response and enhance axonal sprouting in neonatal hypoxic-ischemic brain injury. Stroke 2010; 41: $516-523$.

23 Kuge A, Takemura S, Kokubo Y, Sato S, Goto K, Kayama T. Temporal profile of neurogenesis in the subventricular zone, dentate gyrus and cerebral cortex following transient focal cerebral ischemia. Neurol Res 2009; 31: 969-976.

24 Gerhard A, Schwarz J, Myers R, Wise R, Banati RB. Evolution of microglial activation in patients after ischemic stroke: a [11C](R)-PK11195 PET study. Neuroimage 2005; 24: 591-595.

(c) (i) $\odot$ This work is licensed under a Creative Commons Attribution-NonCommercial-NoDerivs 3.0 Unported License. To view a copy of this license, visit http:// creativecommons.org/licenses/by-nc-nd/3.0/ 